Cómo citar este artículo / Referencia normalizada

E Guijarro, C Santandreu Mascarell, L Canós Darós, R Díez Somavilla, E Babiloni Griñón (2018): "Técnicas multicriterio aplicadas a las redes sociales en el sector público: el uso de Twitter por distintos perfiles de fuerzas y cuerpos de seguridad". Revista Latina de

Comunicación Social, 73, pp. 1412 a 1427.

http://www.revistalatinacs.org/073 paper/1314/73es.html

DOI: $10.4185 /$ RLCS-2018-1314

\title{
Técnicas multicriterio aplicadas a las redes sociales en el sector público: el uso de Twitter por distintos perfiles de fuerzas y cuerpos de seguridad
}

\section{Multicriteria techniques applied to social media in public sector:} the use of Twitter by different law enforcement authorities profiles

\author{
Ester Guijarro [CV] (D) ( $(\mathcal{G})$ Profesora Contratado Doctor. Departamento de \\ Organización de Empresas, Universitat Politècnica de València (España) - \\ esguitar@doe.upv.es
}

Cristina Santandreu Mascarell [CV] (D) (G) Profesora Contratado Doctor. Departamento de Organización de Empresas, Universitat Politècnica de València (España) - crisanma@omp.upv.es

Lourdes Canós Darós [드 (D) (G) Profesora Titular de Universidad. Departamento de Organización de Empresas, Universitat Politècnica de València (España) - loucada@omp.upv.es

Rebeca Díez Somavilla (D) ( $)$ Profesora asociada. Departamento de Comunicación Audiovisual, Documentación e Historia del Arte, Universitat Politècnica de València (España)-rdiez@har.upv.es

Eugenia Babiloni Griñón [CV] (D) (G) Profesora Contratado Doctor. Departamento de Organización de Empresas, Universitat Politècnica de València (España) - mabagri@ doe.upv.es

\begin{abstract}
s
[ES] Introducción: Actualmente las organizaciones públicas están potenciando el uso de las redes sociales como herramienta de comunicación de marketing social, con el fin de influir en el comportamiento de los individuos y lograr un bienestar social. Metodología: Se ha utilizado la técnica multicriterio AHP (Analytical Hierarchical Process) aplicada a cuatro perfiles de Twitter de fuerzas y cuerpos de seguridad de distintos ámbitos territoriales. De este modo, se ha representado el modelo como una jerarquía, se han identificado las variables explicativas del buen uso de las redes sociales como instrumento de comunicación y se han agrupado en clústeres de variables. Posteriormente, se han ponderado tanto las variables como los clústeres mediante el juicio de un experto en comunicación.
\end{abstract}


Resultados: De los clústeres de variables explicativas utilizados para el estudio, "C3. Impacto de la cuenta" se ha revelado como el más importante para el experto. En cuanto a las dimensiones, la más relevante es "Número de seguidores". Por otra parte, los resultados muestran la eficacia en la comunicación de las cuentas de Twitter de los diferentes cuerpos y fuerzas de seguridad analizados. Conclusiones: Este trabajo aporta información de gran utilidad práctica, pues se identifican cuáles son las variables fundamentales para conseguir un uso eficiente en las redes sociales y conseguir el objetivo propuesto de influir en los ciudadanos para lograr un bienestar conjunto. Por otra parte, se abren nuevas líneas de investigación que pueden complementar los resultados obtenidos.

[EN] Introduction: Currently, public organizations are promoting the use of social media as a communication tool for social marketing, in order to influence the behaviour of individuals to achieve social welfare. Methodology: The multi-criteria technique AHP (Analytical Hierarchical Process) has been applied to four Twitter profiles of law enforcement authorities from different territorial areas. Thus, the model has been represented as a hierarchy, the explanatory variables that explain the correct use of social media have been identified and grouped into clusters and both variables and clusters have been weighted using the judgment of a communication expert. Results: Among the clusters of explanatory variables used for the study, "C3. Impact of the profile" has been identified as the most important for the expert. As regards the variables, the most relevant is "Number of followers". On the other hand, the results show the effectiveness of Twitter profiles of the different law enforcement authorities analyzed as a communication tool. Conclusions: This work provides practical information, since it identifies which are the fundamental variables to obtain an efficient use in social media and reach the proposed goal of influencing individuals to achieve a common welfare. Furthermore, further research is opened to complement the results obtained.

\section{Keywords}

[ES] AHP; fuerzas y cuerpos de seguridad; marketing social; multicriterio; sector público; Twitter [EN] AHP; law enforcement authorities; social marketing; multicriteria; public sector; Twitter

\section{Contents}

[ES] 1. Introducción. 2. Metodología. 3. Resultados. 4. Conclusiones. 5. Referencias bibliográficas [EN] 1. Introduction. 2. Methodology. 3. Results. 4. Conclusions. 5. List of references

Artículo traducido por Yuhanny Henares (Traductora académica, Universitat de Barcelona)

\section{Introducción}

El marketing social tiene como finalidad influir en el comportamiento voluntario del público objetivo con el fin de contribuir a mejorar la sociedad. Según Andreasen (1995) el marketing social utiliza técnicas de marketing comercial para el análisis, planteamiento, ejecución y evaluación de programas en pro del beneficio social. Kotler (2002) lo define como la utilización de principios y técnicas para influir sobre una audiencia objetivo para que, de manera voluntaria, acepte, rechace, modifique o abandone un comportamiento en beneficio de individuos, grupos o del conjunto de la sociedad. En los últimos años, tanto este concepto como su ámbito de aplicación han ido evolucionando, surgiendo así distintas definiciones y delimitaciones del mismo. Sin embargo, en la World Social Marketing Conference 2014, Philip Kotler lo define con una sola palabra: bienestar. 
El éxito de un programa de marketing social se medirá en función de su eficacia y pertinencia (Hornik, 2001). A este respecto, la comunicación del mensaje que se desea transmitir juega un papel fundamental en la estrategia de marketing y cobra una especial relevancia definir correctamente los canales de comunicación más eficientes y pertinentes como garantía para alcanzar el objetivo social objeto del mensaje. En el contexto actual, la comunicación es, mayoritariamente, de carácter digital (Patrut, 2015), la cual viene determinada no sólo por el contenido y los usuarios, sino también por las tecnologías existentes (Keïnanen y Kuvalainen, 2015). Internet ha contribuido a potenciar el uso de la comunicación on-line (Jayaram et al., 2015) mientras que las redes sociales se han convertido en una plataforma de comunicación estratégica (Guesagala, 2016) que ha creado desafíos muy interesantes para los profesionales de la comunicación: los mensajes actuales han de ser creativos, innovadores y consistentes digitalmente (Evans et al., 2011).

Las estrategias de comunicación de marketing social en redes buscan crear relaciones reales con su público objetivo (Hanna et al., 2011; Mangold y Faulds, 2009). En este sentido, las redes sociales ofrecen a las organizaciones una herramienta única para construir y mantener relaciones con su público objetivo (Killian y McManus, 2015), proporcionan la oportunidad de establecer y fomentar la participación de los usuarios, la conversación, la colaboración e interacción, así como las conexiones multilaterales (McCann y Barlow, 2015) generando así interrelaciones entre las instituciones públicas y la sociedad (Yi Oh y Kim, 2013). Por lo tanto, en este contexto es muy importante escoger correctamente la red social más adecuada y en este sentido, Twitter parece afianzarse como la más utilizada por las organizaciones en sus estrategias de comunicación del marketing social pues es vista como una red de movilización social, con la que se consigue una gran difusión y viralidad que le permite llegar a una gran mayoría del público general (Arroyo-Almaraz et al. 2018).

En nuestro contexto encontramos ejemplos de la utilización del marketing social, fundamentalmente, en organizaciones no gubernamentales $\mathrm{y}$, sobre todo, en organizaciones públicas que velan por la mejora en cualquier ámbito social. En este sentido, determinar el público objetivo y los canales de comunicación para llegar al mismo juegan un papel fundamental en el éxito de la estrategia de marketing social (Andreasen, 2002). Según Waters y Jamal (2011) las principales organizaciones sin ánimo de lucro estadounidenses usan Twitter para mandar mensajes unidireccionales cuyo contenido es, básicamente, información pública. Twitter permite a los usuarios escribir mensajes cortos (140 caracteres hasta el 7 de noviembre de 2017) que se actualizan constantemente, por lo que existe una comunicación en tiempo real. En esta línea, Lovejoy y Saxton (2012) confirman que la mayor parte de las organizaciones sin ánimo de lucro se decantan por una función informativa más que por una función de creación de comunidad o de acción.

Sin embargo, una pregunta sin respuesta en la literatura es qué debe hacer una organización para que sus mensajes en Twitter sean eficientes, es decir, lleguen a más usuarios susceptibles de ser audiencia objetiva de su estrategia de marketing social y consigan un mayor impacto. En este sentido, llama la atención que a pesar de que un gran número de entidades públicas utilizan Twitter como vía de comunicación social, existe un escaso desarrollo de documentos formales que contribuyan a plantear y entender su correcto funcionamiento. Por tanto, resulta necesario proponer y desarrollar marcos analíticos que permitan reconocer los factores de éxito en el uso de estas tecnologías principalmente en términos de interacción y valoración desde una perspectiva ciudadana (Rojas-Martín y Criado, 2015).

Por lo anteriormente expuesto, este artículo tiene como objetivo proponer un modelo multicriterio que permita analizar el uso de Twitter por cuatro organismos públicos, así como la identificación y valoración de dichos factores de éxito cuando se utiliza para lanzar mensajes de marketing social. Para 
lograr este objetivo, se propone la utilización de la técnica multicriterio discreta AHP (Analytical Hierarchical Process) (Saaty, 1980) que permite obtener las prioridades asociadas a un conjunto de alternativas en escenarios con múltiples actores y criterios (tangibles e intangibles). AHP se ha utilizado en numerosas ocasiones para la resolución de problemas multicriterio sobre marketing (Mishra y Mishra, 2018), bien para el análisis de la estrategia de marketing o alguno de sus componentes (véanse, por ejemplo, Min Hua Lu et al., 1994; Büyüközkan et al. 2016; Kord et al. 2016; Wu y Kao, 2017; Ahmad et al. 2017), bien para estudiar el comportamiento del consumidor (Jain et al. 2015; Leung et al. 2016; entre otros). Sin embargo, hasta donde conocemos, la herramienta AHP no se ha aplicado específicamente a un problema enmarcado en el marketing social, siendo este trabajo pionero en este campo.

La ventaja de utilizar AHP en un contexto de marketing social es que permite incluir, en un mismo modelo, variables cuantitativas, así como cualitativas. De este modo puede analizarse de forma conjunta tanto la actividad que realizan los perfiles escogidos, como el impacto que esta actividad tiene en el resto de la comunidad de usuarios de Twitter. Y es que, desde una óptica de marketing social, lo realmente importante es conocer si el uso que se está haciendo de la red social está llegando a influir en la sociedad, cumpliendo así con su objetivo de bienestar social. Para ello, el modelo incluye el juicio de un experto que valora no sólo cada perfil analizado, sino también cada variable, obteniendo de este modo cuáles son las variables más importantes. Del conjunto de organismos públicos que utilizan Twitter, este trabajo muestra cuatro perfiles del campo de la seguridad ciudadana ya que el uso que le dan a esta red social es exclusivamente para enviar mensajes con un contenido de marketing social, mientras que otros entes públicos también la utilizan para hacer marketing público, turístico o incluso político, escapando así del objeto de este estudio. Concretamente, se analiza el perfil de la Policía Nacional (@policia), Mossos de Esquadra (@mossos), London's Metropolitan Police (@ metpoliceuk) y Europol (@europol_eu).

\section{Metodología}

Para lograr dar respuesta a la pregunta de investigación planteada, se emplea la metodología AHP, que consta de cinco etapas:

\section{Etapa 1. Planteamiento del problema}

El primer paso consiste en definir el objetivo de este caso de estudio, que consiste en determinar qué organismo público utiliza mejor las redes sociales para comunicar mensajes de marketing social. Para ello, es necesario recoger toda la información necesaria del problema de estudio de modo que pueda representarse de la forma más completa posible, incluyendo todas las variables que puedan resultar relevantes. Esta información debe considerar el entorno que rodea al problema, identificar las variables explicativas, las alternativas, así como los agentes involucrados en el proceso para poder, posteriormente, seleccionar aquellas que se incluirán en el modelo. En este paso, se han consultado diversas fuentes con estudios sobre el uso de redes sociales (IAB, 2016; Saavedra et al., 2013) y, más concretamente, del uso de las mismas por parte de organismos públicos (Criado y Rojas-Martín, 2015; Criado et al. 2017). Al estudio de toda esta información permite definir no sólo el objetivo del problema, sino también dos aspectos fundamentales: determinar qué red social escoger y qué muestra representativa de la actividad a valorar.

De entre las diversas plataformas digitales que existen, se ha escogido Twitter por su enfoque abierto y participativo ya que permite una mayor difusión del contenido, así como una mayor facilidad de extracción de datos. Por otro lado, el hecho de que su formato comunicativo esté acotado a tan sólo 
140 caracteres hace atractivo el análisis de su uso para comprobar si es posible lograr comunicar mensajes de índole social, con un efecto positivo para la Administración Pública, siendo tan restrictivo el campo de redacción (Bernhardt et al., 2014).

Planteado el problema y elegida la red social, el siguiente paso es acotar el periodo temporal del análisis. En este caso, se analizan seis meses, comprendidos entre el 1 de octubre de 2017 y el 31 de marzo de 2018. Se considera que es un periodo suficiente para evitar el efecto que puede tener en el resultado el hecho de que suceda un acontecimiento de cualquier índole que afecte a la vida pública y que lleve a incrementar la actividad en Twitter. Existen varias herramientas que permiten obtener datos sobre la actividad de los perfiles en Twitter no siendo los propietarios de los mismos. En este trabajo se utiliza mayoritariamente Twitonomy, debido a la gran cantidad de información que presenta y analiza. Con esta herramienta, el periodo temporal más amplio del que se ha podido tener acceso a los datos ha sido, precisamente, de seis meses. Es importante aclarar que muchas de las herramientas existentes para obtener información sobre perfiles y su actividad acotan la búsqueda por número de tuits. Sin embargo, en este estudio se utiliza un periodo temporal preestablecido ya que la actividad de cada perfil puede diferir y el utilizar sólo el criterio cuantitativo de la actividad para seleccionar la muestra puede hacer que se analicen instantes distintos, sesgando así el resultado.

\section{Etapa 2. Selección de las alternativas comparables}

Dentro del amplio abanico de organismos públicos, el campo de estudio se ha acotado a organismos de las fuerzas y cuerpos de seguridad, ya que es precisamente en este sector donde las redes sociales se utilizan mayoritaria y casi exclusivamente para lanzar mensajes de marketing social. El objetivo de estos mensajes es, precisamente, influenciar en la ciudadanía para lograr un bien social y general. Concretamente se analizan cuatro perfiles, que a su vez cubren distintos ámbitos territoriales: Policía Nacional (@policia) a nivel estatal, Mossos de Esquadra (@mossos) a nivel autonómico, London's Metropolitan Police (@metpoliceuk) a nivel local, pero de una envergadura similar a los anteriores y Europol (@europol_eu) a nivel europeo.

\section{Etapa 3. Selección de las variables explicativas}

El siguiente paso es seleccionar el conjunto de variables con relevancia en el problema propuesto, es decir, aquellas características que explican el buen hacer en el uso de las redes sociales como instrumento de marketing social. Dichas variables explicativas han sido escogidas por un experto en el ámbito de la comunicación en redes sociales y se incluyen tanto variables de carácter cuantitativo como cualitativo. Precisamente, para poder incluir y cuantificar estas últimas se hará uso de la metodología AHP, ya que otras técnicas multicriterio presentan la limitación de ser válidas sólo para variables cuantitativas. Una vez seleccionadas todas las variables explicativas, se agrupan en clústeres según la relación entre las mismas, formando grupos con variables homogéneas entre sí y manteniendo la mayor heterogeneidad posible entre grupos de modo que los clústeres sean independientes entre sí.

En este trabajo se han seleccionado un total de 22 variables agrupadas en 4 clústeres. El primero incluye las variables relacionadas con el entorno social y tecnológico. El segundo recoge las variables explicativas de la propia organización. El tercer clúster reúne todas las variables que hacen relación a la actividad que tienen los perfiles en Twitter. Y, por último, el último clúster incluye las variables relacionadas con el impacto que esa actividad tiene en el resto de la comunidad de Twitter. Nótese que dentro de este clúster aparecen dos sub-clústeres relacionados con los retuits y favoritos recibidos de otros usuarios. La Tabla 1 resume esta información indicando, además, en la última columna, la fuente de información utilizada para poder obtener los datos de cada variable para cada perfil. 


\begin{tabular}{|c|c|c|c|}
\hline \multicolumn{3}{|c|}{ C1. ENTORNO SOCIAL Y TECNOLÓGICO } & FUENTE \\
\hline 1.1 & \multicolumn{2}{|c|}{ Número de habitantes (año 2017) } & INE \\
\hline 1.2 & \multicolumn{2}{|c|}{ Edad media poblacional (año 2017) } & INE \\
\hline 1.3 & \multicolumn{2}{|c|}{ Porcentaje de hogares con acceso a Internet } & INE \\
\hline \multicolumn{3}{|c|}{ C2. ORGANIZACIÓN } & \\
\hline 2.1 & \multicolumn{2}{|c|}{ Plan estratégico de comunicación } & Organización* \\
\hline 2.2 & \multicolumn{2}{|r|}{$\begin{array}{l}\text { Existencia dpto. cuya misión es la difusión a través de } \\
\text { redes sociales }\end{array}$} & Organización* \\
\hline 2.3 & \multicolumn{2}{|c|}{ Grado de experiencia de la organización en Twitter } & Twitonomy \\
\hline \multicolumn{3}{|c|}{ C3. ACTIVIDAD DE LA CUENTA } & \\
\hline 3.1 & \multicolumn{2}{|c|}{ Idioma de publicación } & Metricspot \\
\hline 3.2 & \multicolumn{2}{|c|}{ Números de seguidos } & Twitonomy \\
\hline 3.3 & \multicolumn{2}{|c|}{ Número de tuits que hace el perfil } & Twitonomy \\
\hline 3.4 & \multicolumn{2}{|c|}{ Número de retuits que el perfil hace } & Twitonomy \\
\hline 3.5 & \multicolumn{2}{|c|}{ Número de respuestas que hace el perfil } & Twitonomy \\
\hline 3.6 & \multicolumn{2}{|c|}{ Media tuits/día } & Twitonomy \\
\hline \multicolumn{3}{|c|}{ C4. IMPACTO DE LA CUENTA } & \\
\hline 4.1 & \multicolumn{2}{|c|}{ Número de seguidores } & Twitonomy \\
\hline \multirow[t]{4}{*}{4.2} & \multicolumn{3}{|c|}{ Retuits de otros usuarios } \\
\hline & 4.2 .1 & Número de tuits retuiteados por otros usuarios & Twitonomy \\
\hline & 4.2 .2 & Número de veces que se han retuiteado los tuits & Twitonomy \\
\hline & 4.2 .3 & Media retuits/ tuits & Twitonomy \\
\hline \multirow[t]{4}{*}{4.3} & \multicolumn{3}{|c|}{ Favoritos recibidos de otros usuarios } \\
\hline & 4.3 .1 & $\begin{array}{l}\text { Número de tuits marcados como favoritos por otros } \\
\text { usuarios }\end{array}$ & Twitonomy \\
\hline & 4.3 .2 & $\begin{array}{l}\text { Número de veces marcado como favorito por otros } \\
\text { usuarios }\end{array}$ & Twitonomy \\
\hline & 4.3 .3 & Media favoritos/ tuits & Twitonomy \\
\hline 4.4 & \multicolumn{2}{|r|}{ Eficiencia (retuits y favoritos) / Número total de tuits } & Twitonomy \\
\hline 4.5 & \multicolumn{2}{|c|}{ Hashtags totales } & Twitonomy \\
\hline 4.6 & \multicolumn{2}{|c|}{ Menciones hechas por el perfil } & Tinfoleak \\
\hline
\end{tabular}

*Organización: información publicada de la organización

Tabla 1. Variables explicativas agrupadas en clústeres y fuentes de información.

\section{Etapa 4. Modelización del problema como una jerarquía}

Una vez identificado el objetivo del problema, las alternativas y los criterios, se estructuran formando un árbol jerárquico. De este modo, el nodo superior del árbol representa el objetivo del problema (determinar qué perfil utiliza mejor Twitter como herramienta de comunicación del marketing social), 
en los niveles inferiores se ubican las alternativas (perfiles de Twitter), y se reservan los niveles intermedios para los criterios y subcriterios (variables explicativas agrupadas en clústeres) como se representa en la Figura 1. Todos los elementos de la jerarquía tienen una relación de dependencia unidireccional en sentido ascendente desde el nivel inferior del árbol (hojas) hacia el nodo superior (raíz).

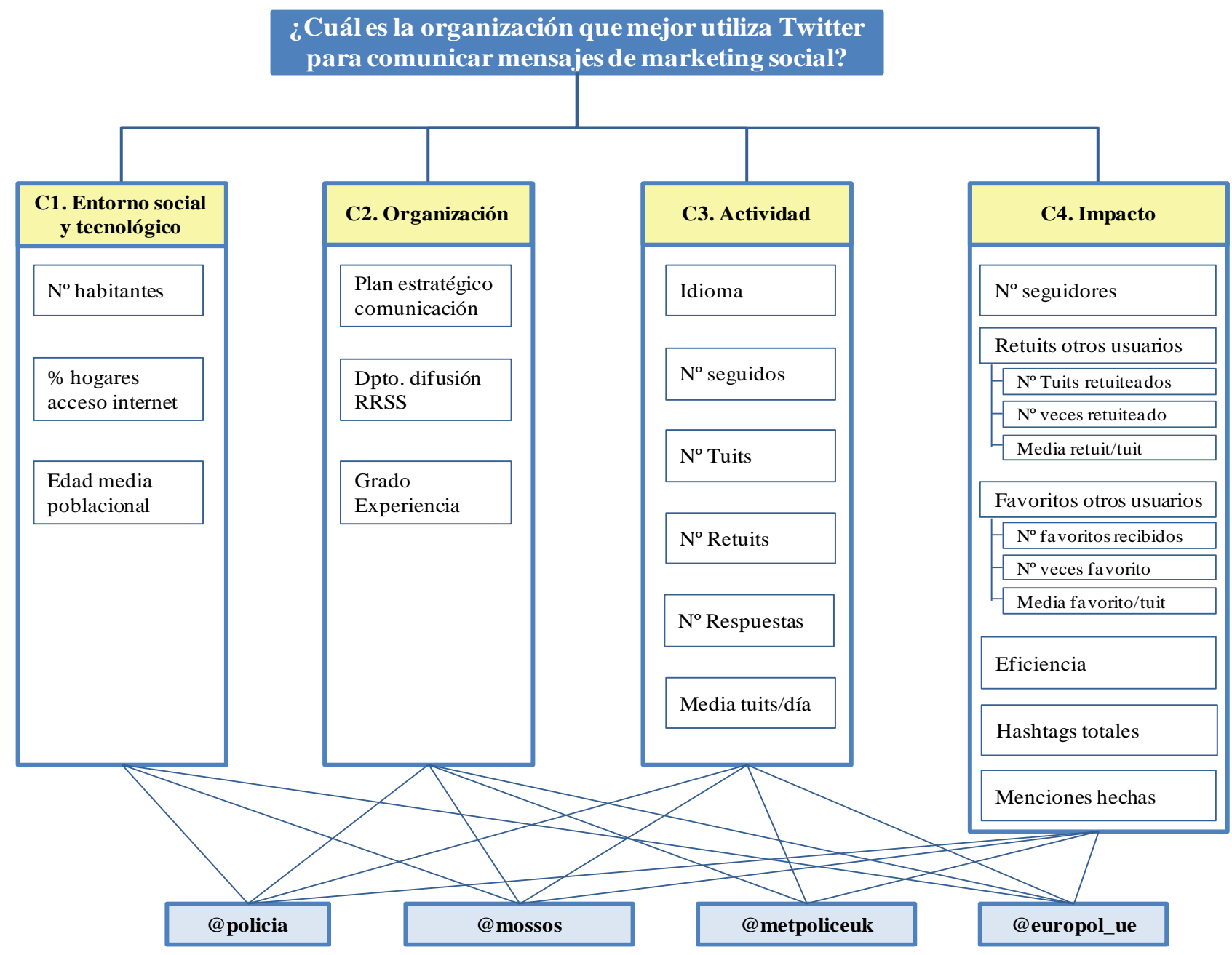

Figura 1. Jerarquía del problema

\section{Etapa 5. Priorización de las alternativas mediante AHP}

En esta etapa se incluye la valoración del experto en comunicación y redes sociales. Para ello, se plantean comparaciones pareadas que permiten cuantificar las variables de carácter cualitativo y se resuelven empleando la escala fundamental de Saaty (ver Tabla 2). De este modo pueden obtenerse, en primer lugar, las prioridades locales de las alternativas y de los criterios. Posteriormente, mediante el cálculo del autovector se obtienen las prioridades locales, que evalúan la importancia relativa que el experto otorga a los elementos que cuelgan de un nodo común respecto a éste; y las prioridades globales, o prioridades de los elementos respecto al objetivo del problema. Por último, aplicando un proceso de agregación de prioridades globales, se extraen las prioridades totales de las alternativas para los diferentes caminos que las unen con la meta, para obtener finalmente el peso o ponderación 
total de cada uno de los perfiles analizados. En el caso de las variables explicativas de tipo cuantitativo se incorporan directamente en el proceso de valoración mediante el cálculo del vector propio por normalización de los valores como porcentaje del total.

\begin{tabular}{|c|l|l|}
\hline Escala numérica & \multicolumn{1}{|c|}{ Escala verbal } & \multicolumn{1}{c|}{ Comentarios } \\
\hline 1 & Igual dominancia & $\begin{array}{l}\text { El criterio A es igual de dominante que el } \\
\text { criterio B. }\end{array}$ \\
\hline 3 & Dominancia moderada & $\begin{array}{l}\text { La experiencia y el juicio favorecen } \\
\text { ligeramente al criterio A sobre el B. }\end{array}$ \\
\hline 5 & Dominancia grande & $\begin{array}{l}\text { La experiencia y el juicio favorecen } \\
\text { fuertemente al criterio A sobre el B. }\end{array}$ \\
\hline 7 & Dominancia muy grande & $\begin{array}{l}\text { El criterio A es mucho más dominante } \\
\text { que el B. }\end{array}$ \\
\hline 9 & Dominancia extrema & $\begin{array}{l}\text { La mayor importancia del criterio A } \\
\text { sobre el B está fuera de toda duda. }\end{array}$ \\
\hline $2,4,6$ y 8 & Valores intermedios entre los anteriores, cuando es necesario matizar. \\
\hline $\begin{array}{c}\text { Recíprocos de } \\
\text { lo anterior }\end{array}$ & $\begin{array}{l}\text { Si el criterio A es de dominancia grande frente al criterio B las notaciones serían } \\
\text { las siguientes: } \\
\text { Criterio A frente a criterio B 5/1 } \\
\text { Criterio B frente a criterio A 1/5 }\end{array}$ \\
\hline
\end{tabular}

Tabla 2. Escala fundamental de comparaciones pareadas de Saaty (1980)

Entre las ventajas de AHP se encuentra la posibilidad de evaluar la consistencia o inconsistencia del experto a la hora de emitir los juicios mediante el cálculo del denominado índice de consistencia (Aguarón y Moreno-Jiménez, 2003; Saaty, 1980). De esta forma, en aquellos casos en que se detecte un nivel de inconsistencia inadmisible, el experto tiene la oportunidad de modificar sus juicios iniciales hasta superar este problema. Existen varias formas de detectar la inconsistencia de una matriz y medir la consistencia de los juicios emitidos. La utilizada en este trabajo consiste en calcular la ratio de consistencia (Saaty, 1980) que se define como el cociente entre el índice de consistencia y el índice de consistencia aleatorio, es decir, mide la relación entre la consistencia alcanzada por la matriz estudiada y el índice de consistencia medio que se alcanzaría si se completara una matriz de la misma dimensión de forma aleatoria utilizando los valores de la escala fundamental de Saaty.

En esta etapa se realizan, pues, los cálculos para cuantificar: (i) las variables cualitativas, (ii) la importancia relativa de cada clúster en el problema y (iii) la importancia relativa de cada variable dentro de su correspondiente clúster, comprobando en cada punto la consistencia de los juicios emitidos por el experto. A modo de ejemplo, se reproducen en la Tabla 3 las comparaciones realizadas por el experto para definir la importancia relativa de las variables explicativas del clúster C3. Actividad de la cuenta, así como el vector propio (VP) obtenido en este caso. El valor de cualquier elemento $a_{i j}$ en la matriz de comparaciones pareadas representa la importancia relativa que el experto otorga al elemento i-ésimo (fila i) frente al elemento j-ésimo (columna j). Nótese que la importancia de elemento j-ésimo frente al elemento i-ésimo es la inversa, de modo que sólo es necesario que el experto conteste la diagonal superior de la matriz. En el caso de comparar cinco elementos, la matriz de comparaciones pareadas queda como: 


$$
\mathbf{A}=\left[\begin{array}{cccc}
w_{1} / w_{1} & w_{1} / w_{2} & \ldots & w_{1} / w_{5} \\
w_{2} / w_{1} & w_{2} / w_{2} & \ldots & w_{2} / w_{5} \\
\vdots & \vdots & \ddots & \vdots \\
w_{5} / w_{1} & w_{5} / w_{2} & \ldots & w_{5} / w_{5}
\end{array}\right]=\left[\begin{array}{cccc}
1 & a_{12} & \ldots & a_{15} \\
a_{21} & 1 & \ldots & a_{25} \\
\vdots & \vdots & \ddots & \vdots \\
a_{51} & a_{52} & \ldots & 1
\end{array}\right]
$$

Después de completar todas las comparaciones pareadas se obtiene el vector de prioridades $\mathbf{w}$, como solución del sistema de ecuaciones $\mathbf{A} \mathbf{w}=\lambda_{\max } \mathbf{w}$, donde $\lambda_{\max }$ es el mayor valor propio de la matriz A y $\mathbf{w}$ es su correspondiente vector propio.

\begin{tabular}{|c|c|c|c|c|c|c|c|}
\hline $\begin{array}{l}\text { C3 - } \\
\text { ACTIVIDAD }\end{array}$ & Idioma & $\begin{array}{c}\mathrm{N}^{\mathrm{o}} \\
\text { Seguidos }\end{array}$ & $\begin{array}{l}\mathrm{N}^{\circ} \\
\text { Tuits }\end{array}$ & $\begin{array}{c}\mathrm{N}^{\mathrm{o}} \\
\text { Retuits }\end{array}$ & $\begin{array}{c}\mathrm{N}^{\mathrm{o}} \\
\text { Respuestas }\end{array}$ & $\begin{array}{l}\text { Media } \\
\text { tuits/día }\end{array}$ & VP \\
\hline Idioma & & 5 & $1 / 7$ & 3 & $1 / 5$ & $1 / 7$ & 0,0646 \\
\hline $\mathrm{N}^{\mathrm{o}}$ Seguidos & & & $1 / 9$ & $1 / 3$ & $1 / 9$ & $1 / 9$ & 0,0221 \\
\hline $\mathrm{N}^{\mathrm{o}}$ Tuits & & & & 7 & 3 & $1 / 3$ & 0,2823 \\
\hline $\mathrm{N}^{\mathrm{o}}$ Retuits & & & & & $1 / 5$ & $1 / 9$ & 0,0377 \\
\hline $\mathrm{N}^{\mathrm{o}}$ Respuestas & & & & & & $1 / 3$ & 0,1703 \\
\hline Media tuits/día & & & & & & & 0,4230 \\
\hline
\end{tabular}

Tabla 3. Matriz de comparación pareada para las variables del clúster C3.Actividad de la cuenta

En este caso de estudio y para facilitar la labor del experto, se le plantean por clústeres una serie de cuestionarios (Figura 2) en el que se le pide que indique con una $\mathrm{X}$ cuánto más importante es el elemento de la izquierda frente al de la derecha. De este modo, el experto no debe cuantificar utilizando la escala de Saaty, sino simplemente marcando en el cuestionario. Posteriormente, se traslada la información que el experto indica en el cuestionario a las matrices de comparación pareada, comprobando en cada caso el índice de consistencia. En aquellos casos en los que se detecta una ratio de consistencia superior al permitido, se le pide al experto que rellene de nuevo el cuestionario, eliminando así las inconsistencias surgidas.

Para el criterio C3.- Actividad de la Cuenta, complete:

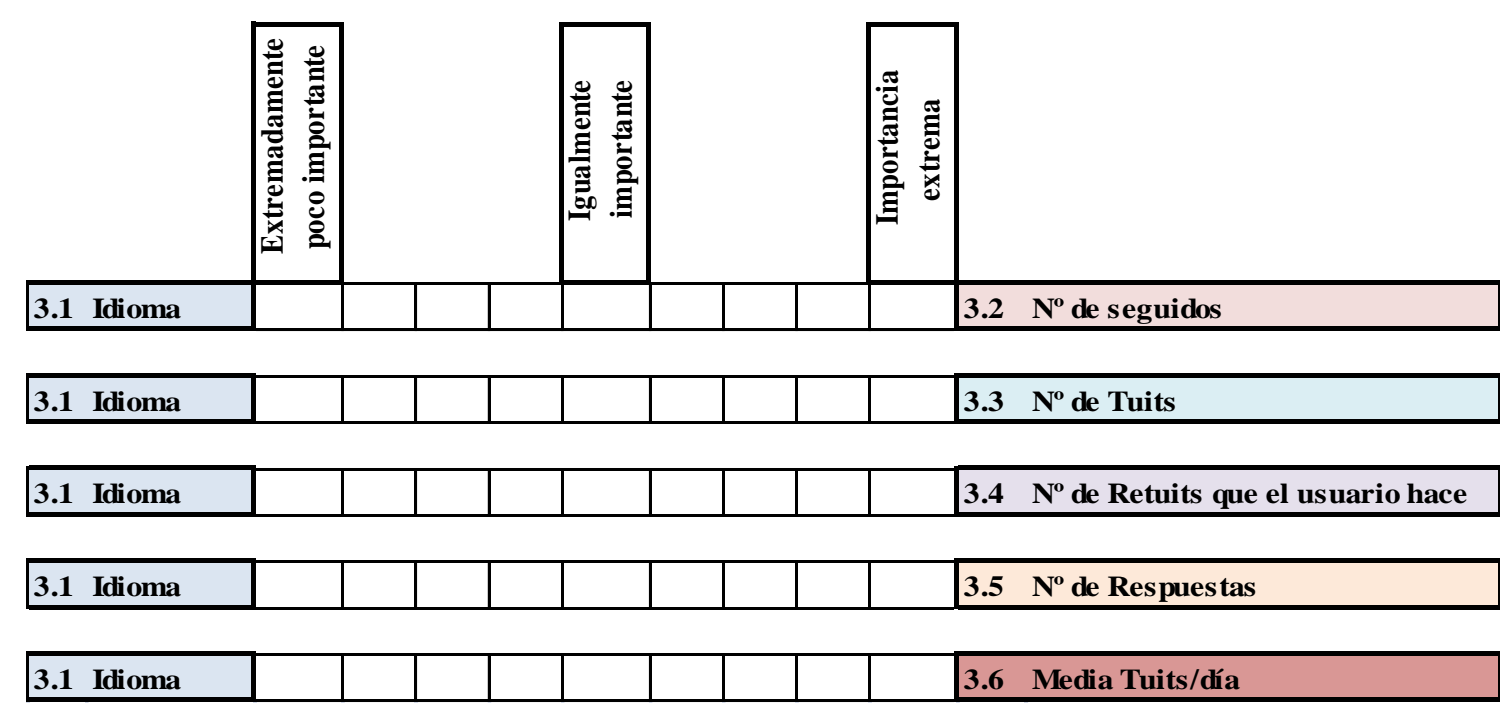

Figura 2. Extracto del cuestionario planteado al experto para determinar la importancia de las variables del clúster C3. Actividad de la cuenta 
Una vez calculados todos los vectores propios, se procede a la agregación para obtener la ponderación final de cada alternativa, así como la ponderación que cada variable explicativa tiene en el problema según los juicios del experto.

\section{Resultados}

Una de las grandes ventajas de utilizar AHP como herramienta de decisión multicriterio es que permite conocer no sólo cuál es la mejor alternativa, sino ver cuál es la importancia relativa que tiene cada variable explicativa dentro de su correspondiente clúster y cada clúster dentro del problema. Destaca, además, que esta información viene determinada por el juicio de un experto en el ámbito de la comunicación a través de redes sociales. En la Tabla 4 se presenta el peso que cada clúster tiene. Puede observarse cómo el clúster más importante según el experto es el de las variables relacionadas con el "C3. Impacto de la cuenta", pues en la comunicación de mensajes de marketing social no sólo es importante lanzar el mensaje, sino que este llegue a la ciudadanía, generando así un impacto y una reacción por parte del resto de usuarios a través de retuits, menciones, etc. Sin embargo, la actividad propiamente dicha del perfil es también importante, porque para tener impacto hace falta lanzar mensajes, de ahí que el segundo clúster en orden de importancia sea el "C2. Actividad", seguido por el "C1. Entorno" y "C2. Organización".

\begin{tabular}{|l|c|}
\hline \multicolumn{1}{|c|}{ Clúster } & \multicolumn{1}{c|}{$\begin{array}{c}\text { Peso } \\
\text { clúster }\end{array}$} \\
\hline $\begin{array}{l}\text { C1. Entorno social y } \\
\text { tecnológico }\end{array}$ & 0,0630 \\
\hline C2. Organización & 0,0630 \\
\hline C3. Actividad del cuenta & 0,1554 \\
\hline C4. Impacto de la cuenta & 0,7186 \\
\hline
\end{tabular}

Tabla 4. Ponderación de los clústeres de variables

En la dimensión de las variables (Tabla 5), la más relevante en el conjunto del modelo es la de "Número de seguidores" con un peso global de 33\%, seguida por el "Número de retuits recibidos" $(12,13 \%)$ y por la "Eficiencia" (11,49\%). Analizando las variables dentro de cada clúster, se observa que, con mucha diferencia, la más importante en "C1. Entorno social y tecnológico" es la "Edad media poblacional" (con un peso del 75,14\%), lo que está directamente relacionado con el público objetivo de los mensajes de marketing social y el canal de difusión utilizado, en este caso, las redes sociales. Sin embargo, esta variable apenas tiene un peso de 4,73\% en el conjunto del modelo. Por su parte, la variable más importante en el clúster "C2. Organización" es la existencia de un "Plan estratégico de difusión" en el que se enmarquen las estrategias y líneas de actuación que debe seguir la organización en el uso de Twitter como herramienta de comunicación de mensajes sociales. No obstante, y como sucedía en el caso anterior, el peso global de esta variable es del 4,23\% del conjunto. En el clúster relacionado con la "C3. Actividad de la cuenta", la variable explicativa más importante es la "Media de tuits diarios" que realice el perfil, lo que indica que es aconsejable hacer un uso constante de las redes para llegar a tener un impacto en la comunidad de usuarios, más que un uso errático en el que algunos días se publique mucha información y otros en cambio la actividad sea mucho menor. Por otro lado, el "Número de tuits" y de "Respuestas que realiza el perfil" son también variables importantes pues son claros indicadores de la actividad que tiene la cuenta, así como su relación con sus seguidores y otros usuarios. Por último, en el clúster "C4. Impacto de la cuenta", la variable más importante es el 
"Número de seguidores" (45,64\%) seguida por el "Número de retuis recibidos " (16,88\%) y la "Eficiencia" (15,99\%), pues como ya se ha indicado estas variables dan información sobre el interés que genera la información publicada por el perfil y su impacto en el resto de la comunidad de usuarios.

\begin{tabular}{|c|c|c|c|}
\hline \multicolumn{2}{|c|}{ Variable } & Peso dentro del clúster & Peso global \\
\hline \multicolumn{4}{|l|}{ C1. ENTORNO } \\
\hline \multicolumn{2}{|l|}{$\mathrm{N}^{\mathrm{o}}$ Habitantes } & 0,0704 & 0,0044 \\
\hline \multicolumn{2}{|c|}{ Edad media poblacional } & 0,7514 & 0,0473 \\
\hline \multicolumn{2}{|c|}{$\%$ Hogares acceso internet } & 0,1782 & 0,0112 \\
\hline \multicolumn{4}{|c|}{ C2. ORGANIZACIÓN } \\
\hline \multicolumn{2}{|c|}{ Plan estratégico difusión } & 0,6716 & 0,0423 \\
\hline \multicolumn{2}{|c|}{ Dpto. difusión RRSS } & 0,0629 & 0,0040 \\
\hline \multicolumn{2}{|l|}{ Grado experiencia } & 0,2654 & 0,0167 \\
\hline \multicolumn{4}{|l|}{ C3. ACTIVIDAD } \\
\hline \multicolumn{2}{|l|}{ Idioma } & 0,0646 & 0,0100 \\
\hline \multicolumn{2}{|l|}{$\mathrm{N}^{\mathrm{o}}$ Seguidos } & 0,0221 & 0,0034 \\
\hline \multicolumn{2}{|l|}{$\mathrm{N}^{\mathrm{o}}$ Tuits } & 0,2823 & 0,0439 \\
\hline \multicolumn{2}{|l|}{$\mathrm{N}^{\mathrm{o}}$ Retuits } & 0,0377 & 0,0059 \\
\hline \multicolumn{2}{|l|}{$\mathrm{N}^{\mathrm{o}}$ Respuestas } & 0,1703 & 0,0265 \\
\hline \multicolumn{2}{|l|}{ Media tuits/día } & 0,4230 & 0,0657 \\
\hline \multicolumn{4}{|l|}{ C4. IMPACTO } \\
\hline \multicolumn{2}{|l|}{$N^{o}$ Seguidores } & 0,4564 & 0,3280 \\
\hline \multirow{3}{*}{ Tuits retuiteados } & $\mathrm{N}^{\mathrm{o}}$ tuits retuiteados & 0,0159 & 0,0114 \\
\hline & $\mathrm{N}^{\circ}$ retuits recibidos & 0,1688 & 0,1213 \\
\hline & Media retuit/tuit & 0,0668 & 0,0479 \\
\hline \multirow{3}{*}{$\begin{array}{l}\text { Tuits marcados } \\
\text { favoritos }\end{array}$} & $\begin{array}{l}\mathrm{N}^{\circ} \text { tuits marcados } \\
\text { favorito }\end{array}$ & 0,0016 & 0,0011 \\
\hline & $\mathrm{N}^{\circ}$ total favoritos & 0,0166 & 0,0120 \\
\hline & Media favorito/tuit & 0,0066 & 0,0047 \\
\hline \multicolumn{2}{|l|}{ Eficiencia } & 0,1599 & 0,1149 \\
\hline \multicolumn{2}{|l|}{ Hashtags } & 0,0438 & 0,0315 \\
\hline \multicolumn{2}{|c|}{ Menciones del perfil } & 0,0637 & 0,0458 \\
\hline
\end{tabular}

Tabla 5. Ponderación de las variables obtenidas mediante matrices de comparación pareada

Agregando los resultados anteriores junto con la ponderación de cada alternativa, se obtiene la ponderación global de cada perfil analizado, información que queda recogida en la Tabla 6. En ella se observa que en primera posición se encuentra la Policía Nacional, seguida por la London's Metropolitan Police, los Mossos de Esquadra y la Europol, respectivamente. Este resultado viene a avalar el buen hacer de la Policía Nacional en materia de comunicación a través de redes sociales. De hecho, no sólo se sitúa en primera posición, sino que la importancia que resulta de la aplicación del modelo es destacadamente mayor que el resto $(45,37 \%)$. 


\begin{tabular}{|l|c|}
\hline \multicolumn{1}{|c|}{ Perfil } & Peso final \\
\hline @policia & 0,4537 \\
\hline @mossos & 0,1966 \\
\hline @metpoliceuk & 0,2396 \\
\hline @ europol_ue & 0,1100 \\
\hline
\end{tabular}

Tabla 6. Ponderación final de los perfiles de Twitter

La Tabla 7 presenta el vector de pesos de cada perfil para cada una de las veintidós variables explicativas. Analizando estos datos, llama la atención que la Policía Nacional es precisamente el único de los cuatro perfiles analizados que no sigue a nadie en Twitter (Tabla 7(b)). Sin embargo, eso no influye en su capacidad de impactar en la comunidad de usuarios. De hecho, los datos muestran que, no sólo es un perfil que tiene una alta actividad en la red social, sino que además, el impacto de sus mensajes es muy elevado, como muestran los datos de Retuits y Favoritos recibidos (Tabla 7(c)(d)).

\begin{tabular}{c|ccc|ccc|} 
& \multicolumn{3}{|c|}{ C1- ENTORNO } & \multicolumn{3}{c|}{ C2- ORGANIZACIÓN } \\
& $\begin{array}{c}\mathbf{N}^{\mathbf{0}} \\
\text { Habitantes poblacional }\end{array}$ & $\begin{array}{c}\text { Edad } \\
\text { Hogares } \\
\text { acceso } \\
\text { internet }\end{array}$ & $\begin{array}{c}\text { Plan } \\
\text { estratégico } \\
\text { difusión }\end{array}$ & $\begin{array}{c}\text { Dpto. } \\
\text { Difusión } \\
\text { RRSS }\end{array}$ & $\begin{array}{c}\text { Grado } \\
\text { experiencia }\end{array}$ \\
\hline @policia & 0,0822 & 0,2895 & 0,2401 & 0,2500 & 0,0500 & 0,2911 \\
@ mossos & 0,0133 & 0,0849 & 0,2418 & 0,2500 & 0,0500 & 0,2615 \\
@metpoliceuk & 0,0015 & 0,0425 & 0,2677 & 0,2500 & 0,4500 & 0,2776 \\
@europol & 0,9030 & 0,5831 & 0,2504 & 0,2500 & 0,4500 & 0,1698 \\
\hline
\end{tabular}

Tabla 7(a). Ponderación relativa de cada perfil (clúster C1. Entorno y C2. Organización)

\begin{tabular}{c|cccccc|} 
& \multicolumn{5}{|c|}{ C3 - ACTIVIDAD } & Nodia \\
& Idioma & $\begin{array}{c}\mathbf{N}^{\mathbf{0}} \\
\text { Seguidos }\end{array}$ & $\mathbf{N}^{\mathbf{o}}$ Tuits & $\mathbf{N}^{\mathbf{0}}$ Retuits & $\begin{array}{c}\mathbf{N}^{\mathbf{2}} \\
\text { Respuestas }\end{array}$ & $\begin{array}{c}\text { Medits/día } \\
\text { tuits }\end{array}$ \\
\hline @policia & 0,2500 & 0,0000 & 0,3531 & 0,2715 & 0,1675 & 0,3530 \\
@mossos & 0,2500 & 0,0450 & 0,2408 & 0,1951 & 0,2884 & 0,2409 \\
@ metpoliceuk & 0,2500 & 0,4599 & 0,3472 & 0,4379 & 0,4836 & 0,3470 \\
@europol & 0,2500 & 0,4951 & 0,0589 & 0,0955 & 0,0604 & 0,0590 \\
\hline
\end{tabular}

Tabla 7(b). Ponderación relativa de cada perfil (clúster C3. Actividad)

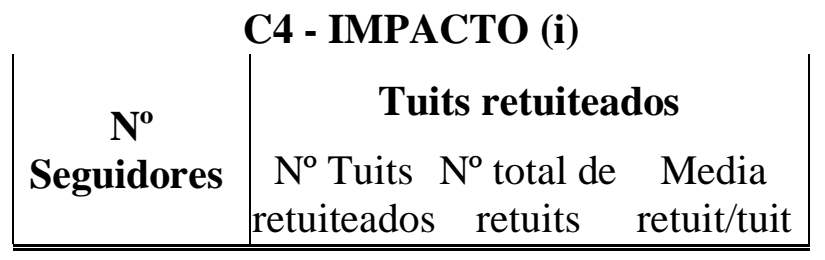




\begin{tabular}{c|cccc}
\hline @ policia & 0,6426 & 0,3623 & 0,5869 & 0,4948 \\
@mossos & 0,1019 & 0,2457 & 0,2358 & 0,2932 \\
@ metpoliceuk & 0,2421 & 0,3394 & 0,1666 & 0,1500 \\
@ europol & 0,0134 & 0,0526 & 0,0107 & 0,0621 \\
\hline
\end{tabular}

Tabla 7(c). Ponderación relativa de cada perfil (clúster C4. Impacto(i))

\begin{tabular}{|c|c|c|c|c|c|c|}
\hline & \multicolumn{6}{|c|}{ C4 - IMPACTO (ii) } \\
\hline & \multicolumn{3}{|c|}{ Tuits marcados favoritos } & \multirow[b]{2}{*}{ Eficiencia } & \multirow[b]{2}{*}{ Hashtags } & \multirow[b]{2}{*}{$\begin{array}{l}\text { Menciones } \\
\text { del perfil }\end{array}$} \\
\hline & $\begin{array}{l}\mathrm{N}^{\mathrm{o}} \text { Tuits } \\
\text { marcados } \\
\text { favorito } \\
\end{array}$ & $\begin{array}{l}\mathrm{N}^{\mathrm{o}} \text { total } \\
\text { favoritos }\end{array}$ & $\begin{array}{l}\text { Media } \\
\text { favorito/tuit }\end{array}$ & & & \\
\hline @policia & 0,3630 & 0,6479 & 0,5514 & 0,2616 & 0,4759 & 0,1861 \\
\hline$@$ mossos & 0,2456 & 0,2782 & 0,3499 & 0,2598 & 0,1406 & 0,4025 \\
\hline @ metpoliceuk & 0,3376 & 0,0674 & 0,0617 & 0,2484 & 0,3043 & 0,2136 \\
\hline @europol & 0,0538 & 0,0065 & 0,0371 & 0,2303 & 0,0792 & 0,1978 \\
\hline
\end{tabular}

Tabla 7(d). Ponderación relativa de cada perfil (clúster C4. Impacto(ii))

\section{Conclusiones}

En este trabajo se utiliza la metodología AHP para analizar el uso que hacen cuatro organismos públicos, en concreto, cuerpos de las fuerzas de seguridad, de las redes sociales para comunicar mensajes de marketing social. Además de poder observar que es el perfil de la Policía Nacional el que obtiene una ponderación destacadamente mayor que el resto, este trabajo aporta información de gran importancia y utilidad práctica. Los resultados de este estudio permiten identificar cuáles son las variables que más importancia tienen a la hora de hacer un uso eficiente de las redes sociales para este fin, de modo que los responsables de la comunicación de mensajes de marketing social de otros organismos públicos pueden utilizar estos resultados para definir una estrategia eficiente que logre, precisamente, el objetivo planteado: influir en los ciudadanos para lograr un bienestar social.

Como futuras líneas de trabajo se plantean básicamente dos: seguir con este estudio, pero utilizando la metodología Analytic Network Process (ANP) desarrollada por Saaty (1996) y que al igual que en la metodología AHP aquí empleada persigue una priorización de las alternativas a partir de comparaciones pareadas entre los elementos del modelo. Sin embargo, con ANP se construye un modelo mucho más complejo y realista que la pura estructura jerárquica del modelo AHP ya que incorpora la posible interdependencia y realimentación entre los distintos elementos que lo componen. De este modo, los problemas de decisión se aproximan mucho más a la realidad ya que incluye las interacciones que se producen entre los elementos del problema, así como entre los agentes del entorno. Por otro lado, se considera muy interesante ampliar y completar este estudio, realizado desde un punto de vista más cuantitativo, con un análisis cualitativo de los mensajes realizados por estos perfiles, dado que la investigación cualitativa ayuda a moderar las comunidades online. Dicha investigación aportaría más información para conocer el plan pragmático (quién habla y desde qué contexto) y, sobre todo, el plano semántico (qué se quiere decir en el mensaje, cuál es el objetivo) que permite distinguir la información relevante. 


\section{Referencias}

Ahmad, F.; Khan, A.A.; Baig, M.M. (2017). Weighting of Marketing Mix elements using fuzzy Analytic Hierarchy Process and Area Based Ranking on Fuzzy Numbers. NED University Journal of Research-Applied Sciences, vol. XIV, no. 2, pp. 35.

Aguaron, J.; Moreno-Jiménez, J.M. (2003). The geometric consistency index: Approximated thresholds. European Journal of Operational rResearch, vol. 147, no.1, pp. 137-145.

Andreasen, A. (1995). Marketing Social Change: Changing Behaviour to promote Health, Social Development and the Environment. San Francisco: Jossey-Bass Publishers.

Andreasen, A.R. (2002). Marketing in the Social Change Marketplace. Journal of Public Policy and Marketing, vol 21, no. 1, pp. 3-13.

Arroyo-Almaraz, I.M.; Calle Mendoza, S.; Van Wyk, C. (2018). La eficacia en la comunicación de las ONGD. El uso de Facebook en campañas de emergencia. Revista Latina de Comunicación Social, 73, 765-789.

Bernhardt, J. M., Alber, J., Gold, R. S. (2014). "A social media primer for professionals: digital dos and don'ts". Health promotion practice, 15(2), 168-172.

Büyüközkan, G.; Mukul, E.; Uztürk, D. (2016). Marketing strategy selection for logistic companies. XIV International Logistics and Supply Chain Congress, December, Izmir (Turquía), pp. 437-445.

Criado, J.I.; Rojas-Martín, F. (2015). Estrategias y realidades en la difusión de las redes sociales en las administraciones públicas. Análisis desde una perspectiva institucionalista. Innovar: Revista de ciencias administrativas y sociales, vol. 25 , no. 57 , pp. 45-62.

Criado, J.I.; Rojas-Martín, F.; Gil-García, J.R. (2017). Enacting social media success in local public administrations: An empirical analysis of organizational, institutional, and contextual factors. International Journal of Public Sector Management, vol. 30, no. 1, pp. 31-47.

Evans, A.; Twormey, J.; Talan, S. (2011). Twitter as a Public Relations Tool. Public Relations Journal, 5(1), Winter.

Guesalaga, R. (2016). The use of social media in sales: Individual and organizational antecedents, and the role of customer engagement in social media. Industrial Marketing Management, 54, 71-79.

Hanna, R.; Rohm, A.; Crittenden, V.L. (2011). We're all connected: The power of the social media ecosystem. Business Horizons, 54, 265-273.

Hornik, R. (2001). Remarks on the Ocassion of the Andreasen Fellowship Lecture. Social Marketing and Health Conference (22 June). Clearwater.

Interactive Advertising Bureau (IAB) (2016). "VII Estudio anual redes sociales de IAB Spain", Madrid: iabspain.

Jain, A.; Kumar, A.; Kumar Dash, M. (2015). Information technology revolution and transition marketing strategies of political parties: analysis through AHP. International Journal of Business Information Systems, vol. 20, issue 1. 
Jayaram, D.; Manrai, A.K.; Manrai, L.A. (2015). Effective use of marketing technology in Eastern Europe: Web analytics, social media, customer analytics, digital campaigns and mobile applications. Journal of Economics, Finance and Administrative Science, 20, 118-132.

Keinänen, H.; Kuivalainen, O. (2015). Antecedents of social media B2B use in industrial marketing context: customers' view. Journal of Business \& Industrial Marketing, 30(6), 711-722.

Killian, G.; McManus, K. (2015). A marketing communications approach for the digital era: Managerial guidelines for social media integration. Business Horizons, 58, 539-549.

Kord, B.; Heidari, Z.; Rigi, F. (2016). Identification, and ranking of marketing strategies in handicraft industries of Fars province Iran with AHP attitude. IIOABJ, vol. 7, 1, pp. 397-404.

Kotler, P.; Roberto, N.; Lee, N. (2002). Social Marketing: Strategies for Changing Public Behaviour. Thousand Oaks: Sage Publications.

Leung, P.P.L.; Wu, C.H.; Ip, W.H.; Ho, G.T.S.; Cho, V.W.S.; Kwong, K.K.Y. (2016). Customer loyalty enhancement of online-tooffline marketing in beauty industry. $4^{\text {th }}$ International Conference on Enterprise Systems (ES), November, Melbourne (Australia).

Lovejoy, K.; Saxton, G.D. (2012). Information, Community, and Action: How Nonprofit Organizations Use Social Media. Journal of Computer-Mediated Communication, 17, 337-353.

Mangold, W.G.; Faulds, D.J. (2009). Social media: The new hybrid element of the promotion mix. Business Horizons, 52, 357-365.

McCann, M.; Barlow, A. (2015). Use and measurement for social media in SMEs. Journal of Small Business and Enterprise Development, 22(2), 273-287.

Min Hua Lu, C.N.; Madu, C.K.; Dena, W. (1994). Integrating QDF, AHP y Benchmarking en marketing strategic. Journal of Business \& Industrial Marketing, vol. 9, issue 1, pp. 41-50.

Mishra, R.; Mishra, O.N. (2018). A hybrid PCA-AHP-Multi-Grade Fuzzy approach to assess marketing-based flexibility. Marketing intelligence \& Planning, vol. 36, issue 2, pp. 213-229.

Patrut, M. (2015). Candidates in the presidential elections in Romania (2014): The use of social media in political marketing. Studies and Scientific Researches. Economics Edition No. 21.

Rojas-Martín, F.; Criado, J.I. (2015). Avanzando en la medición de las redes sociales digitales: una propuesta para su análisis desde una perspectiva institucionalista. Revista de Gestión Pública, vol. Iv, nо. 1, pp. 46-64.

Saaty, T. (1980). The Analytic Hierarchy Process. Pittsburgh: RWS Publications.

Saaty, T. (1996). The Analytic Network Process: decision making with dependence and feedback. Pittsburgh: RWS Publication

Saavedra, F.U.; Rialp Criado, J.; Llonch Andreu, J. (2013). El uso de las redes sociales digitales como herramienta de marketing en el desempeño empresarial. Cuadernos de Administración, vol. 26, no. 47 , pp. 205-231. 
Waters, R.D.; Jamal, J.Y. (2011). Tuit, tuit, tuit: A content analysis of nonprofit organizations' Twitter updates. Public Relations Review, 7, 321-324.

Wu, C.J.; Kao, H. (2017). The marketing project selection using AHP and 0-1 goal programming model. Transylvanian Review, vol. XXV, no. 16.

Yi, M.; Oh, S.; Kim, S. (2013). Comparison of social media use for the U.S an the Korean Governments. Government Information Quarterly, 310-317.

\section{Cómo citar este artículo / Referencia normalizada}

E Guijarro, C Santandreu Mascarell, L Canós Darós, R Díez Somavilla, E Babiloni Griñón (2018): “Técnicas multicriterio aplicadas a las redes sociales en el sector público: el uso de Twitter por distintos perfiles de fuerzas y cuerpos de seguridad”. Revista Latina de Comunicación Social, 73, pp. 1412 a 1427.

http://www.revistalatinacs.org/073paper/1314/73es.html

DOI: $10.4185 /$ RLCS-2018-1314

- En el interior de un texto:

...E Guijarro, C Santandreu Mascarell, L Canós Darós, R Díez Somavilla, E Babiloni Griñón (2018: 1412 a 1427) ...

o

...E Guijarro et al, 2018 (1412 a 1427) ...

Artículo recibido el 7 de mayo de 2018. Aceptado el 18 de mayo.

Publicado el 4 de noviembre de 2018 